\title{
DIRECT AND INVERSE TASK FOR THE OPERATOR STURM-LIOUVILLE TYPE WITH LINEAR DELAY
}

\section{ISMET KALČO, MILENKO PIKULA and FATIH DESTOVIĆ}

Faculty of Polytechnic

University of Zenica

Zenica

Bosnia and Herzegovina

e-mail: hanasim@windowslive.com

Faculty of Philosophy

University of East Sarajevo

Bosnia and Herzegovina

e-mail: pikulam1947@gmail.com

Faculty of Natural Sciences

University of Sarajevo

Sarajevo

Bosnia and Herzegovina

\begin{abstract}
This work is dedicated to solving the direct and inverse spectral task SturmLiouville type with linear delay and dispensed boundary conditions. Under the direct spectral assignment, we mean the construction of solutions $D^{2} y=z^{2} y$. The aim of this paper is to solve the inverse task for the operator $D^{2}$, which to
\end{abstract} 2010 Mathematics Subject Classification: 35Qxx.

Keywords and phrases: characteristic function, asymptotic zero, linear delay, potential, Fourier coefficients.

Received January 17, 2017; Revised February 8, 2017

(C) 2017 Scientific Advances Publishers 
our knowledge is not known in the literature. We propose a new method. This method is based on the transformation of $F$ in such a form that establishes a connection between the Fourier coefficients of a specific set of functions and values of their own operator $D^{2}$, second. In this article, we will assume that $h=0$.

\section{Introduction}

This paper is dedicated to solving the direct and inverse spectral assignment type Sturm-Liouville with linear delay

$$
\tau(x)=\alpha x+\beta,(\alpha, \beta) \in(0,1) \times\left[\frac{(1-\alpha)^{3}}{\alpha^{2}-3 \alpha-3} \beta, \frac{(1-\alpha)^{2}}{2-\alpha} \pi\right),
$$

and partitioned boundary conditions. Let is

$$
q \in L_{2}[0, \pi], \gamma(x)=(1-\alpha) x-\beta, \xi_{1}=\frac{\beta}{1-\alpha} . \text { Therefore } \gamma\left(\xi_{1}\right)=0 .
$$

Studying border assignments $D^{2} y=\lambda y$ given by

$$
\begin{gathered}
-y^{\prime \prime}(x)+q(x) y(\gamma(x))=\lambda y(x) ; \lambda=z^{2}, \\
y(\gamma(x), z) \equiv 0, \quad x \in\left[0, \xi_{1}\right), \\
y^{\prime}(0, z)-h y(0, z)=0, \\
y^{\prime}(\pi, z)+H y(\pi, z)=0 .
\end{gathered}
$$

The potential $q$ and numbers $h, H, \alpha, \beta$ are parameters operator. In particular, if

$\alpha=0$, holds $\gamma(x)=x-\beta$ we have tasks with constant delay expires (see [4], [5], [6], [7]). If $\beta=0$ and $a=1-\alpha$, we have tasks with homogeneously delay (see [8] and [9]). When $\alpha=\beta=0$ assignment detail thrown in the works ([1], [2], [3], and [10]). 


\section{Direct Spectral Assignments}

In this paper, under the direct spectral assignment mean construction solutions $D^{2} y=z^{2} y$ and the construction of the characteristic functions $F$ and its asymptotic zero.

\subsection{Solution task $(1,2,3)$}

Lemma 1. Task $(1,2,3)$ is equivalent to the integral equation type Voltaire

$$
y(x, z)=\cos x z+\frac{h}{z} \sin x z+\frac{1}{z} \int_{\xi_{1}}^{x} q\left(t_{1}\right) \sin z\left(x-t_{1}\right) y\left(\gamma\left(t_{1}\right), z\right) d t_{1} .
$$

Indeed, the method of variation of constants in the Equation (1) using the initial condition (2) and the boundary condition (3) the relation (5) follows directly. We construct a series of functions

$$
\gamma^{k}(x)=\gamma\left(\gamma^{k-1}(x)\right) \text { and series of numbers } \xi_{k}: \gamma^{k}\left(\xi_{k}\right)=0
$$

Lemma 2. There $k_{0} \in N$ are such that it is valid $\xi_{k_{0}}<\pi<\xi_{k_{0}+1}$.

Proof.

$$
\begin{gathered}
\gamma^{1}(x)=\gamma(x)=(1-\alpha) x-\beta=0 \Rightarrow \xi_{1}=\frac{\beta}{1-\alpha} . \\
\gamma^{2}(x)=\gamma(\gamma(x))=(1-\alpha)^{2} x-(1-\alpha) \beta-\beta=0 \Rightarrow \\
\Rightarrow \xi_{2}=\frac{\beta}{(1-\alpha)^{2}}+\frac{\beta}{1-\alpha}=\frac{\beta}{1-\alpha}\left[1+\frac{1}{1-\alpha}\right]=\frac{2-\alpha}{(1-\alpha)^{2}} \beta=\frac{2-\alpha}{1-\alpha} \xi_{1} .
\end{gathered}
$$

Mathematical induction to show valid

$$
\gamma^{k}(x)=(1-\alpha)^{k} x-(1-\alpha)^{k-1} x-\cdots-(1-\alpha) \beta-\beta,
$$

and it follows

$$
\xi_{k}=\gamma^{-1}(0)=\frac{\beta}{1-\alpha}\left[1+\frac{1}{1-\alpha}+\cdots+\frac{1}{(1-\alpha)^{k-1}}\right]=\frac{\beta}{\alpha} \frac{1-(1-\alpha)^{k}}{(1-\alpha)^{k}} .
$$


How to order

$$
\begin{aligned}
& \quad \sum_{k=1}^{\infty} \frac{1}{(1-\alpha)^{k}} \text { diverges, there } k_{0} \in N \text { are such that it is valid } \\
& \xi_{k_{0}}<\pi<\xi_{k_{0}+1} .
\end{aligned}
$$

Therefore,

$$
\left(\xi_{1}, \pi\right]=\bigcup_{k=2}^{k_{0}}\left(\xi_{k-1}, \xi_{k}\right] \cup\left(\xi_{k_{0}}, \pi\right]
$$

In the following, we take the functions

$$
\begin{aligned}
& a_{s c}(x, z)=\int_{\xi_{1}}^{x} q\left(t_{1}\right) \sin \left(x-t_{1}\right) \cos z \gamma\left(t_{1}\right) d t_{1}, \\
& a_{s^{2}}(x, z)=\int_{\xi_{1}}^{x} q\left(t_{1}\right) \sin \left(x-t_{1}\right) \sin z \gamma\left(t_{1}\right) d t_{1},
\end{aligned}
$$

and the recurrent relations

$$
\begin{aligned}
& a_{s^{k} c}(x, z)=\int_{\xi_{k}}^{x} q\left(t_{1}\right) \sin z\left(x-t_{1}\right) a_{s^{k-1} c}\left(\gamma\left(t_{1}\right), z\right) d t_{1}, \quad k>1, \\
& a_{s^{k+1}}(x, z)=\int_{\xi_{k}}^{x} q\left(t_{1}\right) \sin z\left(x-t_{1}\right) a_{s^{k}}\left(\gamma\left(t_{1}\right), z\right) d t_{1}, \quad k>1 .
\end{aligned}
$$

Theorem 1. At a distance $\left(\xi_{l}, \xi_{l+1}\right], l=\overline{1, k_{0}}$ solution of the Equation (5) has the form of

$$
y(x, z)=\cos x z+\frac{h}{z} \sin x z+\sum_{k=1}^{l} \frac{1}{z^{k}}\left[a_{s^{k} c}(x, z)+\frac{h}{z} a_{s^{k+1}}(x, z)\right] .
$$


Proof. For $x \in\left(0, \xi_{1}\right]$ valid $y(x, z)=\cos x z+\frac{h}{z} \sin x z$. For $x \in\left(\xi_{1}, \xi_{2}\right]$, we have

$$
y(x, z)=\cos x z+\frac{h}{z} \sin x z+\frac{1}{z} a_{s c}(x, z)+\frac{h}{z^{2}} a_{s^{2}}(x, z) .
$$

For $x \in\left(\xi_{2}, \xi_{3}\right]$, we have

$$
\begin{aligned}
y(x, z)=\cos x z+\frac{h}{z} \sin x z+\frac{1}{z} a_{s c}(x, z)+\frac{h}{z^{2}} a_{s^{2}}(x, z) & +\frac{1}{z^{2}} a_{s^{2} c^{2}}(x, z) \\
& +\frac{h}{z^{3}} a_{s^{3}}(x, z) .
\end{aligned}
$$

The notion that (7) holds for $l$ and $l+1$. So at a distance $\left(\xi_{l+1}, \xi_{l+2}\right]$ intial solution is exactly the distance $\left(\xi_{l}, \xi_{l+1}\right]$ and it is determined summands

$$
\frac{1}{z^{l+1}}\left[a_{s^{l+1} c}(x, z)+\frac{h}{z} a_{s^{l+2}}(x, z)\right]
$$

which is exactly the (7) for $l+1$.

\subsection{Construction characteristic function}

Construction of characteristic function is based on the solution of the equation at a distance $\left(\xi_{k_{0}}, \pi\right]$ and condition (4). From (7) for $l=k_{0}$ get

$$
\frac{d y}{d x}(x, z)=-z \sin x z+h \cos x z+\sum_{k=1}^{k_{0}} \frac{1}{z^{k-1}}\left[a_{c s^{k-1} c}(x, z)+\frac{h}{z} a_{c s}(x, z)\right]
$$

where is it

$$
\begin{aligned}
& a_{c s^{k-1} c}(x, z)=\int_{\xi_{k}}^{x} q\left(t_{1}\right) \cos z\left(x-t_{1}\right) a_{s^{k-1}}\left(\gamma\left(t_{1}\right), z\right) d t_{1}, \\
& a_{c s^{k}}(x, z)=\int_{\xi_{k}}^{x} q\left(t_{1}\right) \cos z\left(x-t_{1}\right) a_{s^{k}}\left(\gamma\left(t_{1}\right), z\right) d t_{1} .
\end{aligned}
$$


From (7) and (8) on the basis of (4) come to the characteristic function $F(z)$ :

$$
\begin{aligned}
F(z)= & \left(-z+\frac{h H}{z}\right) \sin \pi z+(h+H) \cos \pi z \\
& +\sum_{k=1}^{k_{0}}\left\{\frac{1}{z^{k-1}} a_{c s^{k-1} c}(z)+\frac{1}{z^{k}}\left[h a_{c s^{k}}(z)+H a_{s^{k} c}(z)\right]+\frac{h H}{z^{k+1}} a_{s^{k+1}}(z)\right\},
\end{aligned}
$$

where we have the markings $\pi$.

The function $F$ is the whole function of exponential type with transparent singularity included in the point $z=0$ specified. Within the circle $|z-n|=r_{n}$, where is $r_{n}$ sufficiently small number, there is exactly one zero $z_{n}$ function $F$, which is well known from [3] or [13].

In this study limits may be the case $k_{0}=2$. Therefore

$$
\begin{aligned}
F(z)= & \left(-z+\frac{h H}{z}\right) \sin \pi z+(h+H) \cos \pi z+a_{c^{2}}(z)+\frac{1}{z}\left[h a_{c s}(z)+H a_{s c}(z)\right] \\
& +\frac{h H}{z^{2}} a_{c^{2}}(z)+\frac{1}{z} a_{\mathrm{csc}}(z)+\frac{1}{z^{2}}\left[h a_{c s^{2}}(z)+H a_{s^{2} c^{2}}(z)\right]+\frac{h H}{z^{3}} a_{s^{3}}(z)
\end{aligned}
$$

In the right conditions $k_{0}=2$ limited space allowed for $\beta$ and is $\beta \in\left[\frac{(1-\alpha)^{3}}{\alpha^{2}-3 \alpha-3} \beta, \frac{(1-\alpha)^{2}}{2-\alpha} \pi\right)$.

\subsection{The transformation function $F$}

The finish line of this work is to solve the inverse task for the operator $D^{2}$, which to our knowledge is not known in the literature. Since methods Borg, Geljfand, Marchenko, Crane, Levinson, and other Lejbenzona not apply to the operator with a deviation, we propose a new method. This method is based on the transformation function $F$ in such a 
form that establishes a connection between the Fourier coefficients of a specific set of functions and eigen values of the operator $D^{2}$, second. In this paper, we will assume that $h=0$, which only simplifies technical procedure. First, let us introduce the following codes:

$$
\begin{aligned}
\nu_{-2}^{(1)}= & \frac{1}{2} \xi_{1} ; \nu_{-1}^{(1)}=\frac{1}{2}(\alpha \pi+\beta)=\frac{\tau(\pi)}{2} ; \nu_{0}^{(1)}=\frac{1}{2} \xi_{2}, v_{1}^{(1)}=\frac{\alpha}{2} \pi+l_{1} \xi_{1}, \\
& \nu_{2}^{(1)}=l_{1} \tau(\pi), \nu_{3}^{(1)}=l_{2} \pi-l_{1} \beta, \nu_{4}^{(1)}=l_{1}\left(\pi-\xi_{1}\right), \nu_{5}^{(1)}=\pi-\frac{1}{2} \tau(\pi),
\end{aligned}
$$

where is

$$
l_{1}=1-\frac{\alpha}{2}, \quad l_{2}=1-\alpha+\frac{\alpha^{2}}{2}, \quad l_{1}^{*}=\frac{2}{\alpha}-1 .
$$

Also put

$$
\begin{gathered}
\alpha_{0}=\frac{3-\sqrt{5}}{2}, \underline{\beta}(\alpha)=\frac{(1-\alpha)^{3}}{\alpha^{2}-3 \alpha-3} \pi, \bar{\beta}(\alpha)=\frac{(1-\alpha)^{2}}{2-\alpha} \pi \\
\varphi(\alpha, \beta)=\xi_{2}(\alpha, \beta)-\tau(\pi)=\left[\frac{2-\alpha}{(1-\alpha)^{2}}-1\right] \beta-\alpha \pi .
\end{gathered}
$$

In area $D=\{(\alpha, \beta): \alpha \in(0,1), \beta \in[\underline{\beta}(\alpha), \bar{\beta}(\alpha))\}$ there is a fault $\beta=r(\alpha)$ with a starting point in the $\left(\alpha_{0}, \beta\left(\alpha_{0}\right)\right)$ and end at point $(1,0) \notin D$ such that it is valid

$$
\begin{aligned}
& \quad \varphi(\alpha, r(\alpha))=0, \alpha \in\left[\alpha_{0}, 1\right) . \text { In area } \\
& D_{+}= \\
& \left\{(\alpha, \beta) \in D:\left(\alpha \in\left(0, \alpha_{0}\right) \wedge \beta \in[\underline{\beta}(\alpha), \bar{\beta}(\alpha)) \vee \alpha \in\left(\alpha_{0}, 1\right) \wedge \beta \in(r(\alpha), \bar{\beta}(\alpha))\right)\right\} \\
& \subsetneq D
\end{aligned}
$$

is

$$
0<v_{-2}^{(1)}<v_{-1}^{(1)}<\nu_{0}^{(1)}<v_{1}^{(1)}<\nu_{2}^{(1)}<\nu_{3}^{(1)}<v_{4}^{(1)}<\nu_{5}^{(1)}<\pi .
$$


Definition 1. Function

$$
\left\{\begin{array}{cl}
0, & \theta \in\left[0, \nu_{-2}^{(1)}\right) \cup\left(\nu_{5}^{(1)}, \pi\right], \\
\frac{1}{\alpha} q\left(\frac{2 \theta-\beta}{\alpha}\right)+\frac{1}{2-\alpha} q\left(\frac{2 \theta+\beta}{2-\alpha}\right), & \theta \in\left[\nu_{-2}^{(1)}, \nu_{-1}^{(1)}\right), \\
\frac{1}{2-\alpha} q\left(\frac{2 \theta+\beta}{2-\alpha}\right), & \theta \in\left[\nu_{-1}^{(1)}, \nu_{5}^{(1)}\right]
\end{array}\right.
$$

called transition to function operator $D^{2}$.

We define the following auxiliary functions:

$$
\begin{gathered}
\varphi_{1}^{(1)}(\theta)=\frac{2}{\alpha} \int_{\frac{\theta}{\alpha 1}-\frac{\beta}{\alpha}}^{\frac{2}{\alpha} \theta-l_{1}^{*} \xi_{1}} q\left(t_{1}\right) q\left(\frac{2 \theta-2 \beta-\alpha t_{1}}{\alpha}\right) d t_{1}, \\
\varphi_{1}^{(2)}(\theta)=\frac{2}{\alpha} \int_{\frac{\theta}{\alpha 1}-\frac{\beta}{\alpha}}^{\pi} q\left(t_{1}\right) q\left(\frac{2 \theta-2 \beta-\alpha t_{1}}{\alpha}\right) d t_{1}, \\
\varphi_{2}^{(1)}(\theta)=\frac{2}{\alpha} \int_{\frac{\theta}{l_{2}}+\beta}^{\frac{\theta}{l_{2}}+\xi_{1}} q\left(t_{1}\right) q\left(l_{1}^{*} t_{1}-\frac{2 \beta+2 \theta}{\alpha}\right) d t_{1}, \\
\varphi_{2}^{(2)}(\theta)=\frac{2}{\alpha} \int_{\frac{\theta}{l_{2}}+\beta \frac{l_{1}}{l_{2}}}^{\pi} q\left(t_{1}\right) q\left(l_{1}^{*} t_{1}-\frac{2 \beta+2 \theta}{\alpha}\right) d t_{1}, \\
\varphi_{3}^{(2)}(\theta)=\frac{1}{l_{1}} \int_{\frac{2}{\alpha} \theta-l_{1} \xi_{1}}^{(1)}(\theta)=\frac{\frac{1}{l_{1}}}{\frac{\theta}{l_{1}}+\beta \frac{l_{1}}{l_{2}}} \int_{\frac{2}{\alpha} \theta-l_{1} \xi_{1}}^{\pi} q\left(t_{1}\right) q\left(\frac{\theta}{l_{1}}-\frac{t_{1}}{l_{1}^{*}}\right) d t_{1}, \\
l_{1}
\end{gathered}
$$




$$
\begin{gathered}
\varphi_{4}^{(1)}(\theta)=\frac{1}{l_{1}} \int_{\frac{\theta}{\alpha l_{1}}-\frac{\beta}{\alpha}}^{\frac{\theta}{l_{1}}+\xi_{1}} q\left(t_{1}\right) q\left(t_{1}-\frac{\theta}{l_{1}}\right) d t_{1}, \\
\varphi_{4}^{(2)}(\theta)=\frac{1}{l_{1}} \int_{\frac{\theta}{\alpha l_{1}}-\frac{\beta}{\alpha}}^{\pi} q\left(t_{1}\right) q\left(t_{1}-\frac{\theta}{l_{1}}\right) d t_{1}, \\
K_{1}(\theta, q(\theta))=\left\{\begin{array}{lr}
0, & \theta \in\left[0, v_{0}^{(1)}\right) \cup\left(v_{4}^{(1)}, \pi\right], \\
\varphi_{1}^{(1)}(\theta)-\varphi_{2}^{(1)}(\theta)+\varphi_{3}^{(1)}(\theta)-\varphi_{4}^{(1)}(\theta), & \theta \in\left[v_{0}^{(1)}, v_{1}^{(1)}\right), \\
-\varphi_{2}^{(1)}(\theta)+\varphi_{3}^{(1)}(\theta)-\varphi_{4}^{(1)}(\theta), & \theta \in\left[v_{1}^{(1)}, v_{2}^{(1)}\right), \\
-\varphi_{2}^{(2)}(\theta)-\varphi_{4}^{(2)}(\theta), & \theta \in\left[v_{2}^{(1)}, v_{3}^{(1)}\right),
\end{array}\right. \\
\quad \theta \in\left[v_{3}^{(1)}, v_{4}^{(1)}\right) .
\end{gathered}
$$

Converting the product of trigonometric functions in the forest, by introducing new variables and changing the order of integration we obtain

$$
\begin{aligned}
& a_{c^{2}}(z)=\int_{0}^{\pi} \widetilde{q}^{+}(\theta) \cos z(\pi-2 \theta) d \theta=\widetilde{a}_{c}^{+}(z), \\
& \frac{a_{s c}(z)}{z}=\int_{0}^{\pi} \widetilde{q}^{+}(\theta) \frac{\sin z(\pi-2 \theta)}{z} d \theta=\frac{1}{z} \widetilde{a}_{s}^{+}(z), \\
& \frac{a_{\mathrm{csc}}(z)}{z}=\frac{1}{4} \int_{0}^{\pi} K_{1}(\theta, q(\theta)) \frac{\sin z(\pi-2 \theta)}{z} d \theta, \\
& \frac{a_{s^{2} c}(z)}{z}=-\frac{1}{4} \int_{0}^{\pi} K_{1}(\theta, q(\theta)) \frac{\cos z(\pi-2 \theta)}{z} d \theta .
\end{aligned}
$$


Producing the partial integration in (14), we get

$$
\begin{aligned}
& \frac{a_{\mathrm{csc}}(z)}{z}=\frac{1}{2} \int_{0}^{\pi} \int_{0}^{\theta} K_{1}\left(\theta_{1}, q\left(\theta_{1}\right)\right) d \theta_{1} \cos z(\pi-2 \theta) d \theta, \\
& \frac{a_{s^{2} c}(z)}{z}=\frac{1}{2} \int_{0}^{\pi} \int_{0}^{\theta} K_{1}\left(\theta_{1}, q\left(\theta_{1}\right)\right) d \theta_{1} \sin z(\pi-2 \theta) d \theta .
\end{aligned}
$$

By the relation (15), we have come with the use of the fact that

$$
\lim _{z \rightarrow 0} \frac{a_{s^{2} c}(z)}{z}=0
$$

and then must be

$$
\int_{0}^{\pi} K_{1}(\theta, q(\theta)) d \theta=0 .
$$

So, when $h=0$, the function (11) is transformed into the form of

$$
\begin{aligned}
F(z)= & -z \sin \pi z+H \cos \pi z+\tilde{a}_{c}^{+}(z)+H \frac{\tilde{a}_{s}^{+}(z)}{z} \\
& +\frac{1}{2} \int_{0}^{\pi} \int_{0}^{\theta} K_{1}\left(\theta_{1}, q\left(\theta_{1}\right)\right) d \theta_{1} \cos z(\pi-2 \theta) d \theta \\
& +\frac{H}{2 z} \int_{0}^{\pi} \int_{0}^{\theta} K_{1}\left(\theta_{1}, q\left(\theta_{1}\right)\right) d \theta_{1} \sin z(\pi-2 \theta) d \theta .
\end{aligned}
$$

\subsection{Asymptotic zero function $F$}

Lemma 3. If it is $q \in L_{2}[0, \pi]$ then zero $z_{n}, n \in N$ have the following asymptotic:

$$
z_{n}= \pm\left[n+\left(\frac{H}{\pi}+\frac{1}{\pi} \int_{0}^{\pi} \widetilde{q}^{+}(\theta) \cos 2 n \theta d \theta\right) \frac{1}{n}+O\left(\frac{\left.\int_{0}^{\pi} \tilde{q}^{+}(\theta) \cos 2 n \theta d \theta\right)}{n^{2}}\right](\text { as } n \rightarrow \infty),\right.
$$


where is $\widetilde{q}^{+}$transition function. Indeed, the direct inclusion $z_{n}$ in (16) and (13) follows the accuracy results.

From (16) follow

$$
\lambda_{n}=n^{2}+\frac{2}{\pi} H+\frac{2}{\pi} D^{2} \int_{0}^{\pi} \tilde{q}^{+}(\theta) \cos 2 n \theta d \theta+O\left(\frac{1}{n} \int_{0}^{\pi} \tilde{q}^{+}(\theta) \cos 2 n \theta d \theta\right)
$$

Therefore, eigenvalues operator $D^{2}$ have asymptotic (17).

Note 1. Oscillating member $\tilde{a}_{2 n}^{+}=\frac{2}{\pi} \int_{0}^{\pi} \widetilde{q}^{+}(\theta) \cos 2 n \theta d \theta$ in the (17) is cosine coefficient transition function $\tilde{q}^{+}$that with spaces $\left[0, v_{1}^{(0)}\right)$ and $\left(v_{4}^{(1)}, \pi\right]$ annulled. Because

$$
\tilde{q}^{+}\left(v_{0}^{(1)}\right)=\frac{2}{\alpha(2-\alpha)} \text { and } \tilde{q}^{+}\left(v_{5}^{(1)}\right)=\frac{1}{2-\alpha} q(\pi) \text { we think it's worth }
$$
$q\left(\xi_{1}\right) q(\pi) \neq 0$ which means that the transition function $\widetilde{q}^{+}$in items $v_{0}^{(1)}$ and $\nu_{5}^{(1)}$ has interrupted.

\section{Inverse Spectral Assignments}

Determination of parameters $q, \alpha, \beta, H$ operator $D^{2}$ called solving the inverse spectral assignment. We proceed from the assumption that the two sets of data own values $\lambda_{n j}$ operator $D^{2}$, with the potential $q: q\left(\xi_{1}\right) q(\pi) \neq 0$. From the necessary conditions that we know is true asymptotic

$$
z_{n}=n+c_{j}+\tilde{a}_{2 n}^{+}+O\left(\frac{\tilde{a}_{2 n}^{+}}{2}\right),(\text { as } n \rightarrow \infty), j=1,2,
$$

where are $c_{j}$ and $\tilde{a}_{2 n}^{+}$cosine Fourier coefficients of a function that is reversed at intervals $\left[0, \mu_{1}\right)$ and $\left(\mu_{2}, \pi\right]$ where are $\mu_{1}$ and $\mu_{2}$ known numbers and $\mu_{1}<\mu_{2}$. 
From $\lim _{n \rightarrow \infty}\left(\lambda_{n j}-n^{2}\right)=c_{j}, j=1,2$, we obtain $H_{j}=\frac{\pi}{2} c_{j}$. Still, we are celebrating $\frac{\beta}{2(1-\alpha)}=\mu_{1}$ and $\pi-\frac{1}{2}(\alpha \pi+\beta)=\mu_{2}$ to we obtain the parameters $\alpha$ and $\beta$ of the equation. Thus, the parameters $H_{j}, j=1,2$. and $\alpha$ and $\beta$ are obtained uniformly.

\subsection{Basic identity}

According Adamar's theorems obtain representation of $F$ by their zero $\pm \sqrt{\lambda_{n j}}, n \in N_{0}, j=1,2$ in which

$$
F_{j}(z)=\pi \lambda_{0 j} \prod_{n=1}^{\infty} \frac{\lambda_{n j}}{n^{2}}\left(1-\frac{z^{2}}{\lambda_{0 j}}\right) \prod_{n=1}^{\infty}\left(1-\frac{z^{2}}{\lambda_{n j}}\right),
$$

numbers $\alpha$ and $\beta$ are as defined. In the future we will assume that the following applies:

$$
\begin{gathered}
\alpha \in\left(0, \frac{3-\sqrt{5}}{2}\right) \wedge \beta \in\left[\frac{(1-\alpha)^{3}}{\alpha^{3}-3 \alpha+3} \pi, \frac{(1-\alpha)^{2}}{2-\alpha} \pi\right), \text { or } \\
\alpha \in\left(\frac{3-\sqrt{5}}{2}, 1\right) \wedge \beta \in\left(r(\alpha), \frac{(1-\alpha)^{2}}{2-\alpha} \pi\right), \text { where is } \\
{\left[\frac{2-\alpha}{(1-\alpha)^{2}}-1\right] r(\alpha)-\alpha \pi=0, \quad \alpha \in\left(\frac{3-\sqrt{5}}{2}, 1\right) .}
\end{gathered}
$$

In this way, we can conclude that the other functions on the expression (18) must be of the form (15), i.e., $k_{0}=2$. First, we put

$$
\begin{aligned}
& a_{c}^{(1)}(z)=\int_{0}^{\pi}\left(\int_{0}^{\theta} K_{1}\left(\theta_{1}, q(0,1) d \theta\right) \cos z(\pi-2 \theta) d \theta,\right. \\
& a_{s}^{(1)}(z)=\int_{0}^{\pi}\left(\int_{0}^{\theta} K_{1}\left(\theta_{1}, q(\theta, 1) d \theta_{1}\right) \sin z(\pi-2 \theta) d \theta .\right.
\end{aligned}
$$


We establish basic identities

$$
\begin{aligned}
& A(z)=\frac{H_{2} F_{1}(z)-H_{1} F_{2}(z)}{H_{2}-H_{1}}+z \sin \pi z, \\
& B(z)=z \frac{F_{2}(z)-F_{1}(z)}{H_{2}-H_{1}} z-z \cos \pi z .
\end{aligned}
$$

From (19) we come to the system

$$
\begin{aligned}
& \tilde{a}_{c}^{+}(z)+\frac{1}{2} a_{c}^{(1)}(z)=A(z), \\
& \tilde{a}_{s}^{+}(z)+\frac{1}{2} \widetilde{a}_{s}^{(1)}(z)=B(z), \quad z \in C .
\end{aligned}
$$

System identity (20) is equivalent infinite system

$$
\begin{aligned}
& \frac{2}{\pi} \tilde{a}_{c}^{+}(m)+\frac{2}{\pi} \frac{1}{2} a_{c}^{(1)}(m)=\frac{2}{\pi} A(m), \quad m \in N \cup\{0\}, \\
& \frac{2}{\pi} \tilde{a}_{s}^{+}(m)+\frac{2}{\pi} \frac{1}{2} a_{s}^{(1)}(m), \quad m \in N .
\end{aligned}
$$

Because

$$
\begin{gathered}
\frac{2}{\pi} \widetilde{a}_{c}^{+}(m)=(-1)^{m} \frac{2}{\pi} \int_{o}^{\pi} \widetilde{q}^{+}(\theta) \cos 2 m \theta d \theta=(-1)^{m} \widetilde{a}_{2 m}^{+}, \\
\frac{2}{\pi} \widetilde{a}_{s}^{+}(m)=(-1)^{m+1} \frac{2}{\pi} \int_{o}^{\pi} \widetilde{q}^{+}(\theta) \sin 2 m \theta d \theta=(-1)^{m+1} \widetilde{b}_{2 m}^{+}, \\
\frac{2}{\pi} a_{c}^{(1)}(m)=(-1)^{m} \frac{2}{\pi} \int_{0}^{\pi}\left(\int_{0}^{\theta} K_{1}\left(\theta_{1}, q\left(\theta_{1}\right) d \theta_{1}\right) \cos 2 m \theta d \theta=(-1)^{m} a_{2 m}^{(1)}\right. \\
\frac{2}{\pi} a_{s}^{(1)}(m)=(-1)^{m+1} \frac{2}{\pi} \int_{0}^{\pi}\left(\int_{0}^{\theta} K_{1}\left(\theta_{1}, q\left(\theta_{1}\right) d \theta_{1}\right) \sin 2 m \theta d \theta=(-1)^{m+1} b_{2 m}^{(1)} .\right.
\end{gathered}
$$


And leading mark

$$
\begin{aligned}
\frac{2}{\pi} A(m)(-1)^{m} & =A_{2 m}, \frac{2}{\pi} B(m)(-1)^{m+1}=B_{2 m} \text { from (21) we get } \\
& \widetilde{\mathrm{a}}_{2 m}^{+}+\frac{1}{2} a_{2 m}^{(1)}=A_{2 m}, m \in N_{0}, \\
& \widetilde{b}_{2 m}^{+}+\frac{1}{2} b_{2 m}^{(1)}=B_{2 m}, m \in N .
\end{aligned}
$$

From (19) proves to be true $A_{2 m} \rightarrow 0, B_{2 m} \rightarrow 0, m \rightarrow 0$. So arrays $\left\{A_{2 m}, B_{2 m}\right\}$ represent the Fourier coefficients of a function $f \in L_{2}[0, \pi]$. Multiplying (22) with $\cos 2 m x^{*} i \sin 2 m x^{*}, x^{*} \in[0, \pi]$, after summarizing come to the equation

$$
\widetilde{q}^{+}\left(x^{*}\right)+\frac{1}{2} \int_{0}^{x^{*}} K_{1}(\theta, q(\theta)) d \theta=f\left(x^{*}\right)
$$

Transformation of the transition function $\tilde{q}^{+}$and function $q$ is the tool that we used the method of determining the solutions reduce to solving integral equations of type Volterra. From (11') and (13), we conclude that the Equation (23) breaks up in different forms at intervals $\left[\nu_{-1}^{(1)}, v_{0}^{(1)}\right]$ and $\left[v_{4}^{(1)}, \nu_{5}^{(1)}\right]$ kernel $K_{1}$ is reversed and the Equation (23) becomes

$$
\widetilde{q}^{+}(x)=f(x)
$$

For

$$
x^{*} \in\left[\nu_{-1}^{(1)}, v_{5}^{(1)}\right] \text { valid } \tilde{q}^{+}\left(x^{*}\right)=\frac{1}{2-\alpha} q\left(\frac{2 x^{*}+\beta}{2-\alpha}\right) .
$$

Let's

$$
x_{1}=\frac{\alpha \pi+2 \beta}{2-\alpha}, \quad x_{2}=\frac{\alpha^{2}-3 \alpha+3}{(2-\alpha)(1-\alpha)} \beta, \quad x_{3}=\pi-\frac{\beta}{(2-\alpha)(1-\alpha)},
$$


and

$$
\tilde{f}(x)=(2-\alpha) f\left(\frac{1}{2}[(2-\alpha) x-\beta]\right) .
$$

When out of (231) following $q(x)=\tilde{f}(x), x \in\left[x_{1}, x_{2}\right] \cup\left[x_{3}, \pi\right]$.

On the segment $\left[\nu_{3}^{(1)}, v_{4}^{(1)}\right]$ valid

$$
K_{1}(\theta, q(\theta))=-\left(\varphi_{2}^{(2)}(\theta)+\varphi_{4}^{(2)}(\theta)\right)
$$

so we get

$$
\frac{1}{2-\alpha} q\left(\frac{2 x^{*}+\beta}{2-\alpha}\right)-\frac{1}{2} \int_{\nu_{3}^{(1)}}^{x^{*}}\left(\varphi_{2}^{(2)}(\theta)+\varphi_{4}^{(2)}(\theta)\right) d \theta=f\left(x^{*}\right) .
$$

From (12), we have

$$
\int_{\nu_{3}^{(1)}}^{x^{*}} \varphi_{2}^{(2)}(\theta) d \theta=\frac{2}{\alpha} \int_{\nu_{3}^{(1)}}^{x^{*}} \int_{\frac{\theta}{l_{2}}+\beta \frac{l_{1}}{l_{2}}}^{\pi} q\left(t_{1}\right) q\left(\frac{(2-\alpha)-t_{1} 2 \beta+2 \theta}{\alpha}\right) d t_{1} d t .
$$

By changing the order of integration and the replacement of variables

$$
\frac{(2-\alpha) t_{1}-2 \beta-2 \theta}{\alpha}=t_{2}
$$

we have

$$
\int_{\nu_{3}^{(1)}}^{x^{*}} \varphi_{2}^{(2)}(\theta) d \theta=\int_{\mu_{1}^{(1)}}^{\xi_{1}+\frac{1}{l_{1}} x^{*}} q\left(t_{1}\right)\left(\int_{\alpha \xi_{1}}^{l_{1}^{*} t_{1}-\frac{2 l_{2}}{\alpha} \pi-\beta} q\left(t_{2}\right) d t_{2}\right) d t_{1}
$$

Similar to the previous valid

$$
\int_{\nu_{3}^{(1)}}^{x^{*}} \varphi_{4}^{(2)}(\theta) d \theta=\int_{\mu_{2}^{(1)}}^{-\frac{\beta}{\alpha l_{1}^{2}}+\frac{x^{*}}{\alpha l_{1}}} q\left(t_{1}\right)\left(\int_{(1-\alpha) t_{1}-\frac{\beta}{l_{1}^{2}}}^{\frac{l_{2}}{l_{1}} \pi-\beta} q\left(t_{2}\right) d t_{2}\right) d t_{1}
$$


where is

$$
\mu_{2}^{(1)}=\frac{\pi}{\alpha}-\frac{\beta}{\alpha}-\frac{\beta}{\alpha l_{1}^{2}}, \quad \mu_{1}^{(1)}=\frac{l_{2}}{l_{1}} \pi+\alpha \xi_{1}
$$

Functions

$$
\psi_{1}^{(1)}\left(t_{1}\right)=\int_{\alpha \xi_{1}}^{l_{1}^{*} t_{1}-\mu_{3}^{(1)}} q\left(t_{2}\right) d t_{2}, \quad \mu_{3}^{(1)}=\frac{2 l_{2} \pi}{\alpha}+\beta
$$

and

$$
\psi_{2}^{(1)}\left(t_{1}\right)=\int_{(1-\alpha) t_{1}-\frac{\beta}{l_{1}^{2}}}^{\frac{l_{2}}{l_{1}} \pi-\beta} q\left(t_{2}\right) d t_{2}
$$

are absolutely continuous on fraud

$$
\left[\mu_{1}^{(1)}, \xi_{1}+\frac{1}{l_{1}} x^{*}\right],\left[\mu_{2}^{(1)},-\frac{\beta}{a l_{1}^{2}}+\frac{x^{*}}{a l_{1}}\right] \text { and } x^{*} \in\left[\nu_{3}^{(1)}, v_{4}^{(1)}\right] \text {. }
$$

So, these segments are limited and have performed almost everywhere

Let's

$$
x=\frac{2 x^{*}+\beta}{2-\alpha}, x_{4}=\frac{l_{2}}{l_{1}} \pi+\frac{\alpha \beta}{2 l_{1}}, x_{5}=\pi-\frac{\xi_{1}}{l_{1}^{*}}, x_{6}=\frac{\beta}{\alpha}\left(\frac{1}{2 l_{1}}+\frac{1}{l_{2}^{2}}\right) .
$$

When (23) becomes

$$
q(x)=\tilde{f}(x)+\int_{\mu_{1}^{(1)}}^{x+\frac{\xi_{1}}{2 l_{1}}} q\left(t_{1}\right)(2-\alpha) \psi_{1}^{(1)}\left(t_{1}\right) d t_{1}+\int_{\mu_{2}^{(1)}}^{\frac{1}{\alpha} x-x_{6}} q\left(t_{1}\right)(2-\alpha) \psi_{2}^{(1)}\left(t_{1}\right) d t_{1} .
$$


Let's $\underline{t_{1}}=\min \left\{\mu_{2}^{(1)}, \mu_{2}^{(2)}\right\}$ and $s_{1} x+s_{2}=\max \left\{x+\frac{\xi_{1}}{2 l_{1}}, \frac{1}{\alpha} x-x_{6}\right\}$,

$$
\psi\left(t_{1}\right)=(2-\alpha) \psi_{1}^{(1)}\left(t_{1}\right)+(2-\alpha) \psi_{2}^{(1)}\left(t_{1}\right), \quad h(x)=q(x)-\tilde{f}(x) .
$$

When $\left(\Phi_{3}\right)$ becomes

$$
h(x)=\int_{t_{1}}^{s_{1} x+s_{2}}\left(h\left(t_{1}\right)+\tilde{f}\left(t_{1}\right)\right) \psi\left(t_{1}\right) d t_{1} .
$$

$$
\text { Operator } A(h)=\int_{t_{1}}^{s_{1} x+s_{2}}\left(h\left(t_{1}\right)+\widetilde{f}\left(t_{1}\right)\right) \psi\left(t_{1}\right) d t_{1} \text { with some degree of }
$$

contraction operator and a uniform fixed point. This provides the solution for $q$ at a distance $\left[x_{4}, x_{3}\right]$, where is $x_{4}=\frac{l_{2}}{l_{1}} \pi-\frac{1-\alpha}{2-\alpha} \beta$. With intervals $\left[v_{0}^{(1)}, v_{1}^{(1)}\right],\left[v_{1}^{(1)}, v_{2}^{(1)}\right]$, and $\left[v_{2}^{(1)}, v_{3}^{(1)}\right]$, the solution was prepared by the same procedure as in interval $\left[\nu_{3}^{(1)}, v_{4}^{(1)}\right]$.

Theorem. There is a single partition segments $\left[\xi_{1}, \pi\right]$ and $\left[\nu_{-2}^{(1)}, \nu_{5}^{(1)}\right]$ such that

$$
\left[\xi_{1}, \pi\right]=\bigcup_{k=0}^{\infty}\left[x_{k+1}, x_{k}\right], x_{0}=\pi, x_{\infty}=\xi_{1}
$$

and

$$
\left[\nu_{-2}^{(1)}, \nu_{5}^{(1)}\right]=\bigcup_{k=0}^{\infty}\left[x_{k+1}^{*}\right], x_{0}^{*}=v_{5}^{(1)}, x_{\infty}=v_{-2}^{(1)}, x_{1}^{*}=v_{-1}^{(1)} .
$$


Proof. By using the function $x^{*}=\frac{\alpha x+\beta}{2}$ point $\pi$ exceeding the point $v_{-1}^{(1)}$ and using the tool $x^{*}=\frac{(2-\alpha) x-\beta}{2}$ point $x_{1}=\frac{\alpha \pi+2 \beta}{2-\alpha}$ exceeding the point $\nu_{-1}^{(1)}$ because $x_{1}=\frac{2\left(\frac{\alpha \pi+\beta}{2}\right)+\beta}{2-\alpha}=\frac{\alpha \pi+2 \beta}{2-\alpha}$. Further,

$$
\frac{\alpha x_{1}+\beta}{2}=\frac{1}{2}\left(\frac{\alpha^{2} \pi}{2-\alpha}+\frac{2 \alpha \beta}{2-\alpha}+\beta\right)=x_{2}^{*}
$$

and is

$$
x_{2}=\frac{\left(\frac{\alpha^{2} \pi}{2-\alpha}+\frac{2 \alpha \beta}{2-\alpha}+\beta+\beta\right)}{2-\alpha}=\frac{\alpha^{2} \pi}{(2-\alpha)^{2}}+\frac{2 \alpha \beta}{(2-\alpha)^{2}}+\frac{2 \beta}{2-\alpha} .
$$

Also,

$$
\frac{\alpha x_{2}+\beta}{2}=\frac{1}{2}\left[\frac{\alpha^{3} \pi}{(2-\alpha)^{2}}+\frac{2 \alpha^{2} \pi}{(2-\alpha)^{2}}+\frac{2 \beta}{2-\alpha}+\beta\right]=x_{3}^{*}
$$

and

$$
x_{l}=\left(\frac{\alpha}{2-\alpha}\right)^{l} \pi+\frac{2 \beta}{2-\alpha}\left[\left(\frac{\alpha}{2-\alpha}\right)^{l-1}+\left(\frac{\alpha}{2-\alpha}\right)^{l-2}+\cdots+\frac{\alpha}{2-\alpha}+1\right] .
$$

Directly checking that the formula $\left(23_{2}\right)$ is true when using in (232) instead of $l, l+1$ put and the formula are valid for every $l \in N$.

Moreover valid

$$
\begin{gathered}
\lim _{l \rightarrow \infty} x_{l}=\lim _{l \rightarrow \infty}\left(\frac{\alpha}{2-\alpha}\right)^{l} \pi+\frac{2 \beta}{2-\alpha} \lim _{l \rightarrow \infty}\left[1+\frac{\alpha}{2-\alpha}+\cdots+\left(\frac{\alpha}{2-\alpha}\right)^{l-1}\right] \\
=\frac{2 \beta}{2-\alpha} \lim _{l \rightarrow \infty} \frac{1-\left(\frac{\alpha}{2-\alpha}\right)^{l}}{1-\frac{\alpha}{2-\alpha}}=\frac{\beta}{1-\alpha}=\xi_{1}=x_{\infty} .
\end{gathered}
$$


Like checking that applies

$$
\lim _{l \rightarrow \infty} x_{l}^{*}=\nu_{-2}^{(1)}=\frac{1}{2} \xi_{1} .
$$

This theorem evidence.

Effect 1: While variable $x$ lies in the segment $\left[x_{k+1}, x_{k}\right], k=0,1,2, \ldots$ when valid

$$
\frac{(2-\alpha) x-\beta}{2} \in\left[x_{k+1}^{*}, x_{k}^{*}\right] \text {, and } \frac{\alpha x+\beta}{2} \in\left[x_{k+2}^{*}, x_{k+1}^{*}\right] .
$$

Effect 2: Since the solution of the Equation (23) defined on $\left[x_{1}, \pi\right]$ stops finding potential to $\left[\xi_{1}, x_{1}\right)$. Equation (23) at a distance $\left[v_{-2}^{(1)}, \nu_{-1}^{(1)}\right]$ has the form

$$
\begin{gathered}
\frac{1}{\alpha} q\left(\frac{2 x^{*}-\beta}{\alpha}\right) \pm \frac{1}{2-\alpha} q\left(\frac{2 x^{*}+\beta}{2-\alpha}\right)=f\left(x^{*}\right) \\
x=\frac{2 x^{*}+\beta}{2-\alpha} \in\left[x_{k+2}, x_{k+1}\right] \Rightarrow \frac{2 x^{*}-\beta}{\alpha}=\frac{2-\alpha}{\alpha} x-\frac{2 \beta}{\alpha} \in\left[x_{k+1}, x_{k}\right], \\
k=0,1,2, \ldots
\end{gathered}
$$

Therefore,

$$
q(x)=-\frac{2-\alpha}{\alpha} q\left(\frac{2-\alpha}{\alpha} x-\frac{2 \beta}{\alpha}\right)+f\left(\frac{(2-\alpha) x-\beta}{2}\right) .
$$

If it is $x \in\left[x_{k+1}, x_{k}\right], k=1,2, \ldots$ when it is $\frac{2-\alpha}{\alpha} x-\frac{2 \beta}{\alpha} \in\left[x_{k}, x_{k-1}\right]$.

For $k=0$, we determine the potential $q$ in the previous treatments to the right side (233) is known. Relation (233) yields the potential $q$ on the segment $\left[x_{2}, x_{1}\right]$. Successive potential is determined on each segment $\left[x_{k+1}, x_{k}\right], k=1,2, \ldots$ Finally, this procedure gives potential on $\left[\xi_{1}, x_{1}\right]$. Thus we have proved the main result: 
Theorem 3. Some were given two sets of eigenvalues $\lambda_{n j}, j=1,2$. $n \in N_{0}$ operator $D^{2}(q, \alpha, \beta, 0, H)$ with potential $q: \exists \mu_{1} \in(0, \pi): q\left(\xi_{1}\right)$ $q(\pi) \neq 0$

$$
(\alpha, \beta) \in(0,1) \times(\underline{\beta}(\alpha), \bar{\beta}(\alpha)) ; \underline{\beta}(\alpha)=\frac{-(1-\alpha)^{3}}{\alpha^{2}-3 \alpha-3} \pi, \bar{\beta}(\alpha)=(1-\alpha) \pi
$$

and if $\lambda_{n j}$ him have asymptotic (17') whereby $\tilde{a}_{2 n}{ }^{+}$series of cosine Fourier coefficients function $\widetilde{q}^{+} \in L_{2}[0, \pi]: \widetilde{q}^{+}(x) x \in\left[0, \mu_{1}\right) \cup\left(\mu_{2}, \pi\right], \mu_{1}<\mu_{2}, a$ numbers $\mu_{1}, \mu_{2}$ are known. Then the operator $D^{2}$ is known.

\section{References}

[1] G. Freiling and V. Yurko, Inverse Sturm-Liouville Problems and their Applications, Nova Science Publishers, Inc. Huntington, New York, 2008.

[2] M. G. Fasimov and B. M. Levitan, Determination of a differential equation by two spectra, UMN. (1964)-T(19). No 2. (116). 3-63.

[3] I. M. Gelfand and B. M. Levitan, Determination of a differential equation by its function spektralno. Izv. AN SSSR Ser.Maт-(1951).T.15.-S. 309-360.

[4] Göran Borg, Eine Umkehrung der Strum-Liouvilleschen Eigenwertaufgabe, Acta Math. 78-(1946), 1-96.

[5] M. Pikula, A differential operator of Sturm-Liouville problem with delay of two spectra, Bulletin Mathematica 43 (1991), 159-171.

[6] M. Pikula and T. Marjanovi, The construction of the small potential for an equation of Sturm-Liouville type with constant delay, Mathematical Conference in Pristin (1996), 135-141.

[7] R. I Lazovic and M. Pikula, Regularized trace of the operator applied to solving inverse problems, Radovi Matematicki, 2002.

[8] M. Pikula, On the determination equation of a differential with variable delay equation, Mathematica Montisnigri VI (1996).

[9] V. Vladicic, Doctoral thesis, University of East Sarajrevo, 2013. god.

[10] M. Pikula and I. Kalco, Eigenvalues operator Sturm-type Liouville with variable delay type of $x-\tau(x)$, Proceedings of the Second Mathematical Conference of the Republic of Srpska, (2012), 73-85. 
[11] M. Pikula and I. Kalco, Design solutions border task with linear delay, Proceedings of the Third Mathematical Conference of the Republic of Srpska, (2013).

[12] I. Kalco, Construction solutions differential operator type Sturm -Liouville border task with linear delay, Bulletin of the international mathematical virtual institute ISSN (p) 2303-4874, ISSN (o) 2303-4955, 0.951.

[13] I. Kalco, M. Pikula and V. Miletic, Relations between the Fourier coefficients of transition function operator Sturm-type Liuvila with linear delay and own values of these operators, Proceedings of the Fourth Mathematical Conference of the Republic of Srpska, (2014), 383-394. 